\title{
Non-traditional markers of metabolic risk in prepubertal children with different levels of cardiorespiratory fitness
}

\author{
Francisco Jesús Llorente-Cantarero', Juan Luis Pérez-Navero², Juan de Dios \\ Benitez-Sillero', Maria Carmen Muñoz-Villanueva ${ }^{3}$, Manuel Guillén-del Castillo' and \\ Mercedes Gil-Campos ${ }^{2, *}$ \\ 'Department of Corporal Expression, Faculty of Education, University of Cordoba, Córdoba, Spain: ${ }^{2}$ Department \\ of Pediatrics, University Reina Sofia Hospital, Maimonides Institute for Biomedical Research (IMIBIC), Av de \\ Menéndez Pidal, s/n 14004, Córdoba, Spain: ${ }^{3}$ Unit of Methodology in Investigation, IMIBIC, Córdoba, Spain
}

Submitted 23 May 2011: Accepted 8 December 2011: First published online 16 January 2012

\begin{abstract}
Objective: To assess classical and non-classical metabolic risk biomarkers in prepubertal children with different levels of cardiorespiratory fitness (CRF).

Design: CRF was assessed by the $20 \mathrm{~m}$ shuttle run test. To estimate physical activity, participants were observed while engaged in an after-school programme. Additionally, a short test based on a validated questionnaire was used to obtain information about physical activity practice and sedentary habits. Anthropometric parameters, blood pressure, and classical and non-traditional metabolic risk biomarkers - plasma lipid profile, glucose and insulin, homeostasis model assessment-insulin resistance index (HOMA-IR), plasma uric acid, transaminases and C-reactive protein (CRP) - were measured.

Setting: The study was conducted in local elementary schools in Córdoba, Spain. Subjects: One hundred and forty-one healthy children (eighty-eight boys, fiftythree girls) aged 7-12 years, in Tanner stage I, were recruited. They were divided into two groups after they performed the $20 \mathrm{~m}$ shuttle run test: equal or higher cardiovascular fitness (EHCF) group and low cardiovascular fitness (LCF) group. Results: The LCF group displayed significantly higher TAG $(P=0 \cdot 004)$ and lower HDL cholesterol levels $(P=0 \cdot 001)$, as well as significantly lower values for the non-traditional lipid marker apo-A1 $(P=0 \cdot 001)$ compared with the EHCF group. The LCF children displayed higher plasma glucose $(P=0.003)$ and insulin levels, higher HOMA-IR scores $(P<0 \cdot 001)$ and higher plasma uric acid and CRP levels $(P<0 \cdot 05)$. After adjustment for BMI, age and sex, no statistically significant differences were found between groups for the biomarkers analysed.

Conclusions: The study provides new information to understand the role not only of weight status but also of the level of CRF on the metabolic health profile of prepubertal children.
\end{abstract}

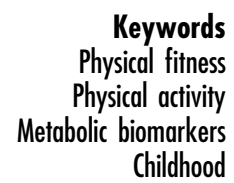

Keywords

Physical activity

Childhood
The metabolic syndrome (MetS) refers to the clustering of cardiovascular risk factors driven by peripheral insulin resistance. Data from recent studies involving a population of children and adolescents indicate that the prevalence of MetS varies between 3\% and $12 \%{ }^{(1)}$. Suggestions for future research include establishing which individual components of the MetS cluster confer the greatest risk on future morbidity in children. There is no universally accepted definition of MetS in childhood ${ }^{(2)}$ but for investigating associations between cardiorespiratory fitness (CRF) and metabolic risk, a total risk score has been calculated in various studies as the sum of $Z$-scores for the single risk factors defining $\mathrm{MetS}^{(3)}$.

However, the International Diabetes Federation consensus defines MetS in children and adolescents as central obesity plus any two of the following classical parameters: (i) raised TAG levels, (ii) reduced HDL cholesterol (HDL-C) levels, (iii) hypertension and (iv) elevated fasting plasma glucose ${ }^{(4)}$. Moreover, some non-classical factors such as plasma uric acid elevation have been associated with features of insulin resistance; indeed, it has been suggested that uric acid could be a key link for the diagnosis of MetS in obese prepubertal children ${ }^{(5)}$. The homeostasis model assessment-insulin resistance index (HOMA-IR) and levels of total cholesterol, LDL cholesterol (LDL-C), apo-A1 and apo- $\mathrm{B}^{(5-7)}$, transaminases ${ }^{(8)}$ and $\mathrm{C}$-reactive protein $(\mathrm{CRP})^{(9)}$ are also altered in children with MetS, but they are considered non-traditional features of MetS.

Going from moderate to high levels of CRF has consistently been associated with a lower risk of developing 
MetS, CVD or type 2 diabetes $^{(10)}$. CRF involves a set of health or skill-related attributes with a strong genetic component that remains relatively static, needing some time to change, and it is assessed using a battery of field tests. Nevertheless, variability in CRF is known to influence metabolic status ${ }^{(3)}$. Physical activity (PA) is often used interchangeably with energy expenditure and physical fitness and has been defined as any bodily movement produced by skeletal muscles which results in energy expenditure. Some authors suggest that CRF and PA may affect metabolic risk but the relationship is weak and they may act through different pathways ${ }^{(11,12)}$.

In children, some studies have shown that CRF is independently associated with clustered metabolic risk factors $^{(11,13)}$. Non-traditional factors have been also researched in children in relation to $\mathrm{CRF}^{(8,14)}$. Nevertheless, further research is needed to ascertain whether changes in fitness in the general population of children predict changes in cardiovascular risk profile and whether fitness and fatness independently influence metabolic risk ${ }^{(8,15)}$. Thus the aim of the present study was to assess classical and non-classical metabolic risk biomarkers, including insulin resistance, plasma lipid profile, uric acid, liver transaminases and CRP, in prepubertal healthy children with different levels of CRF.

\section{Experimental methods}

\section{Participants and design}

A group of 141 healthy children, eighty-eight boys $(\leq 10$ years, $n 52 ;>10$ years, $n 36$ ) and fifty-three girls (all $\leq 10$ years), at prepubertal stage was selected from local elementary schools in Córdoba, Spain. Children were asked to perform a $20 \mathrm{~m}$ shuttle run test (20-mSRT) in order to evaluate their CRF.

Inclusion criteria were age 7-12 years and prepubertal stage (Tanner stage I), as validated by appropriate plasma sex hormone levels. Exclusion criteria were as follows: presence of pubertal development, disease, long periods of rest after illness, use of any medication that alters blood pressure or glucose or lipid metabolism, consumption of any special diet and failure to get the same record reached in the first attempt in the 20-mSRT ${ }^{(16)}$. Written informed consent was obtained from parents or legal guardians and the study was approved by an institutional ethics committee at the University Reina Sofia Hospital.

The validated scale developed by Olds et al. ${ }^{(17)}$ was used to measure CRF after the 20-mSRT. The test performances are expressed as mean and standard deviation relative to all children of similar age and sex from all countries. In the present study, children were split into two groups according to the methodology used in previous studies $^{(18,19)}$ : (i) the children recording a score equal to or greater than the average reference value (seventy-five participants) were assigned to the group designated 'equal or higher cardiovascular fitness' (EHCF); and (ii) those with less-than-average scores (sixty-six participants) were assigned to the group 'low cardiovascular fitness' (LCF).

Furthermore, children were divided into quintiles after estimating their $\mathrm{V}_{\mathrm{O} \text { max }}$ obtained by cross-referencing the final level and shuttle number completed, using the formula developed by Leger et al. ${ }^{(20)}(Y, \mathrm{ml} / \mathrm{kg}$ body weight per $\mathrm{min})$, from the speed $(X, \mathrm{~km} / \mathrm{h})$ corresponding to that stage (speed $=8+0.5$ stage no.) and age ( $A$, years): $Y=31 \cdot 025+3 \cdot 238 X-3 \cdot 248 A+0 \cdot 1536 A X . \quad \mathrm{V}_{\mathrm{O} 2 \max }$ correlates oxygen consumption with exercise duration, and estimated relative $\mathrm{V}_{\mathrm{O} \text { max }}$ and extrapolated absolute $\mathrm{V}_{\mathrm{O} 2 \mathrm{max}}$ are used to determine fitness. Thus top and bottom groups were compared.

\section{Pbysical examination and measurements}

Anamnesis and a physical examination were assessed by paediatricians despite illness in all of the children. Sexual maturity was observed by physical examination according to the Tanner five-stage scale ${ }^{(21)}$.

Weight and height were measured by standard techniques, using a beam balance and a precision stadiometer (SC700; Seca, Hamburg, Germany), with participants lightly dressed and barefooted. BMI was calculated as weight $(\mathrm{kg}) /[\text { height }(\mathrm{m})]^{2}$. Waist circumference was measured in duplicate with an inelastic tape according to standardized methods. These anthropometric measurements were compared with Spanish reference standards ${ }^{(22,23)}$ and with the age- and sex-specific cut-off points proposed by Cole et al. ${ }^{(24)}$ to define obesity. Thus we ensured that most of the children of the study were healthy.

Systolic and diastolic blood pressures (BP) were measured in the right arm in a sitting position, using a random-zero sphygmomanometer (Dinamap V-100; GE Healthcare, Spain) after the participants had rested without changing position for at least $5 \mathrm{~min}$.

Children were observed while engaged in an after-school programme at least three times weekly for at least 1 year to estimate PA. Additionally, a short test based on the validated questionnaire of the National Institute of Child Health and Human Development ${ }^{(25)}$ was used for both groups to obtain information about PA practice and sedentary habits.

\section{Evaluation of cardiorespiratory fitness}

Standardized Eurofit battery tests ${ }^{(20,26)}$ were performed to evaluate fitness; the 20-mSRT was used to assess CRF, and upper and lower body strength were also evaluated.

The 20-mSRT required participants to run back and forth between two lines set $20 \mathrm{~m}$ apart. Running speed started at $8.5 \mathrm{~km} / \mathrm{h}$ and increased by $0.5 \mathrm{~km} / \mathrm{h}$ each minute, reaching $18.0 \mathrm{~km} / \mathrm{h}$ at minute 20 . Running speed cues were indicated by signals emitted by a commercially available CD-ROM. Participants were allowed to voluntarily withdraw from the test after being verbally encouraged to maximally perform during each assessment. The test finished when the participant failed to reach the finishing lines concurrent with the audio signals on two consecutive occasions ${ }^{(27)}$. 
Upper-body muscular strength was assessed by means of the handgrip strength and the bent arm hang tests with a digital hand dynamometer (Takei TKK Q4-5110, precision $0 \cdot 1 \mathrm{~kg}$ and range 5 to $100 \mathrm{~kg}$; Takei Scientific Instruments Co., Ltd, Niigata, Japan) and another test evaluating the number of abdominals performed within 30 s. Lower-body muscular strength was assessed by using the standing broad jump test.

\section{Sampling}

Baseline fasting blood samples were obtained from all children using an indwelling venous line to draw a $3 \mathrm{ml}$ sample for the measurement of plasma glucose and insulin levels, lipid profiles and uric acid levels.

\section{Biochemical analysis}

Fasting gonadotrophins and sex hormones - follicle stimulating hormone $(\mathrm{CV}=3.6 \%)$, luteinizing hormone $(\mathrm{CV}=3 \cdot 1 \%)$, testosterone $(\mathrm{CV}=2 \%)$ and oestradiol $(\mathrm{CV}=1 \cdot 8 \%)$ - were measured by chemiluminescence using an automatic analyser (Architect I4000; Abbott Laboratories, Abbott Park, IL, USA) to validate that the children selected by clinical signs and Tanner stage were truly prepubertal.

Glucose was analysed using the glucose oxidase method in an automatic analyser $(\mathrm{CV}=1 \%)$. Plasma TAG $(\mathrm{CV}=$ $1.5 \%)$, total cholesterol $(\mathrm{CV}=0.9 \%)$, HDL-C $(\mathrm{CV}=0.8 \%)$, LDL-C $(\mathrm{CV}=1.5 \%)$, apo-A1 $(\mathrm{CV}=1.7 \%)$, apo-B $(\mathrm{CV}=2.6$ $\%)$, uric acid $(\mathrm{CV}=1.9 \%)$, aspartate aminotransferase $(\mathrm{CV}=1 \cdot 7 \%)$, alanine aminotransferase $(\mathrm{CV}=3 \%)$ and $\mathrm{CRP}$ were also measured using an automatic analyser (Accelerator APS system, Architect-c16000; Abbott Laboratories). $\mathrm{CRP}(\mathrm{CV}=4 \%)$ was measured by particle-enhanced turbidimetric immunoassay (Dade Behring Inc., Deerfield, IL, USA). Plasma insulin was analysed by RIA with an automatic analyser for microparticles (Axsym; Abbott Laboratories). Insulin resistance was calculated by means of HOMA-IR, defined by the equation: HOMA-IR $=$ [fasting glucose $(\mathrm{mmol} / \mathrm{l}) \times$ fasting insulin $(\mu \mathrm{U} / \mathrm{ml})] / 22 \cdot 5$.

The criteria using classical features agreed by the International Diabetes Federation in $2007^{(4)}$ were used in the present study to estimate the presence of MetS in the sample of prepubertal children.

\section{Statistical analysis}

Data were expressed as means and standard deviations. Normal distribution of data was assessed by the ShapiroWilk test. Homogeneity of variances was estimated using the Levene test. Comparison of means between groups for continuous variables with normal distribution was done using Student's $t$ test for unpaired samples and for those with an asymmetric distribution by the Mann-Whitney $U$ test. The $\chi^{2}$ test was applied for comparison of proportions. Comparisons between the two groups of children after tests were performed using analysis of covariance after adjusting for age, sex and BMI. Correlations between variables were assessed using Pearson's test.

Cohen typified differences $(d)$ in the studied parameters between children in the top and bottom quintiles of fitness and effect size correlation were calculated in order to quantify the magnitude of the difference (effect size).

The statistical software package PASW ${ }^{\circledR}$ Statistics 18 (SPSS Inc., Chicago, IL, USA) was used for all statistical analyses.

\section{Results}

Anthropometric and BP measurements and PA performance between groups of children with different levels of CRF are shown in Table 1. BMI and waist circumference values were significantly higher in the LCF group than in the EHCF group but none of these children were obese. There were no differences in BP levels between both groups.

For the Eurofit physical test battery, the EHCF group displayed significantly higher results in the abdominals test and the horizontal jump test; no between-group differences were observed in the dynamometry test. Most children (72\%) in the EHCF group practised PA regularly in an after-school programme (Table 1). A positive significant correlation was observed between CRF and PA $(r=0.346 ; P<0 \cdot 001)$.

Results for classical MetS biochemical parameters were as follows: the LCF group displayed significantly higher TAG and lower HDL-C levels, as well as significantly lower values for the non-traditional lipid marker apo-A1 (Fig. 1). The LCF group also displayed significantly higher plasma glucose (Fig. 2(a)) and insulin (Fig. 2(b)) and HOMA-IR scores (Fig. 2(c)).

Table 1 Anthropometric parameters, blood pressure and physical activity as a function of cardiorespiratory fitness level in healthy prepubertal children ( $n$ 141) aged 7-12 years, Córdoba, Spain

\begin{tabular}{|c|c|c|c|c|c|}
\hline \multirow[b]{2}{*}{ Variable } & \multicolumn{4}{|c|}{ EHCF group $(n 75)$ LCF group $(n 66)$} & \multirow[b]{2}{*}{$P^{\star}$} \\
\hline & Mean & SD & Mean & SD & \\
\hline Age (years) & $9 \cdot 57$ & $1 \cdot 20$ & $9 \cdot 68$ & 1.09 & 0.5708 \\
\hline Weight $(\mathrm{kg})$ & $38 \cdot 35$ & $9 \cdot 58$ & $45 \cdot 89$ & $12 \cdot 54$ & $<0.0001$ \\
\hline Height $(\mathrm{cm})$ & $142 \cdot 33$ & $9 \cdot 51$ & $144 \cdot 74$ & $8 \cdot 85$ & $0 \cdot 1229$ \\
\hline BMI $\left(\mathrm{kg} / \mathrm{m}^{2}\right)$ & $18 \cdot 69$ & $2 \cdot 89$ & $21 \cdot 55$ & 3.99 & $<0.0001$ \\
\hline WC (cm) & $63 \cdot 20$ & $7 \cdot 64$ & $71 \cdot 21$ & $11 \cdot 56$ & $<0.0001$ \\
\hline SBP $(\mathrm{mmHg})$ & $120 \cdot 00$ & $10 \cdot 36$ & $123 \cdot 25$ & $16 \cdot 09$ & $0 \cdot 1527$ \\
\hline $\mathrm{DBP}(\mathrm{mmHg})$ & $66 \cdot 94$ & $9 \cdot 14$ & $67 \cdot 32$ & $10 \cdot 53$ & $0 \cdot 8213$ \\
\hline 20-mSRT (periods) & $4 \cdot 91$ & $1 \cdot 79$ & $2 \cdot 12$ & $0 \cdot 87$ & $<0.0001$ \\
\hline Abdominals (no.) & $14 \cdot 55$ & $5 \cdot 52$ & 11.94 & $5 \cdot 22$ & 0.0047 \\
\hline $\mathrm{HJ}(\mathrm{m})$ & $1 \cdot 35$ & $0 \cdot 19$ & $1 \cdot 16$ & $0 \cdot 17$ & $<0.0001$ \\
\hline RHD (kg) & $16 \cdot 00$ & $4 \cdot 47$ & $16 \cdot 90$ & $5 \cdot 12$ & 0.2734 \\
\hline LHD (kg) & $15 \cdot 16$ & $4 \cdot 23$ & $16 \cdot 34$ & $6 \cdot 22$ & $0 \cdot 1869$ \\
\hline PPA (\%) & 72 & & 42 & & $0.0007 \dagger$ \\
\hline
\end{tabular}

EHCF, equal or higher cardiovascular fitness; LCF, low cardiovascular fitness; WC, waist circumference; SBP, systolic blood pressure; DBP, diastolic blood pressure; $20-\mathrm{mSRT}, 20 \mathrm{~m}$ shuttle run test; abdominals, abdominal exercises; HJ, horizontal jump; RHD, right hand dynamometry; LHD, left hand dynamometry; PPA, performed physical activity.

* Statistical significance after application of a Student $t$ test to data expressed as mean and SD.

tStatistical significance after application of a $\chi^{2}$ test to data expressed as a percentage. 


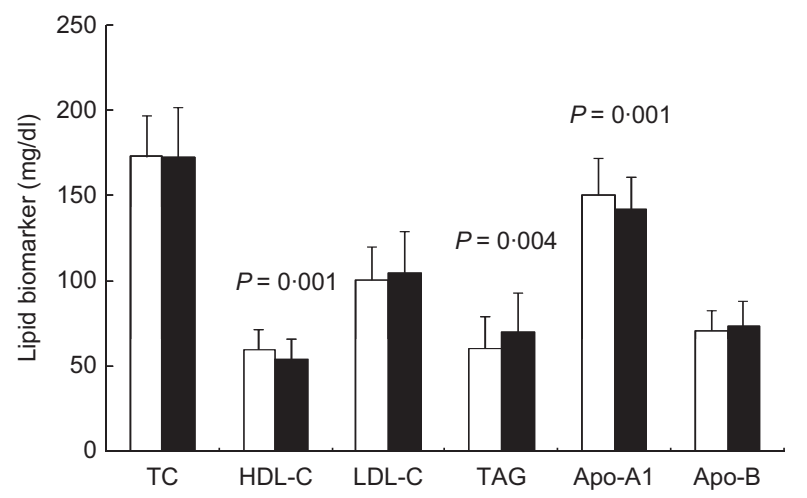

Fig. 1 Lipid biomarkers (TC, total cholesterol; HDL-C, HDL cholesterol; LDL-C, LDL cholesterol) in healthy prepubertal children ( $n$ 141) aged 7-12 years, Córdoba, Spain, according to cardiorespiratory fitness ( $\square$, equal or higher cardiovascular fitness (EHCF) group; $\square$, low cardiovascular fitness (LCF) group). Values are means and standard deviations represented by vertical bars; $P$ values indicate statistical significance after application of a Student $t$ test to the data

(a)

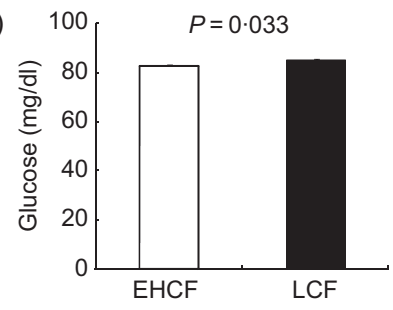

(b)

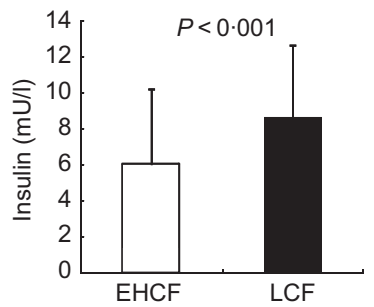

(c)

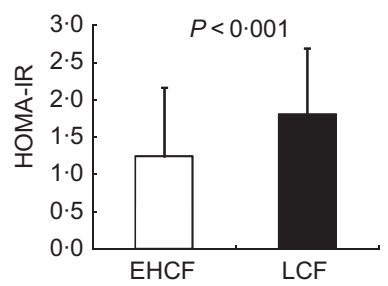

Fig. 2 Insulin resistance biomarkers in healthy prepubertal children (n 141) aged 7-12 years, Córdoba, Spain, according to cardiorespiratory fitness ( $\square$, equal or higher cardiovascular fitness (EHCF) group; $\square$, low cardiovascular fitness (LCF) group): (a) plasma glucose; (b) plasma insulin; (c) homeostasis model assessment-insulin resistance index (HOMA-IR). Values are means and standard deviations represented by vertical bars; $P$ values indicate statistical significance after application of a Student $t$ test to the data

With regard to non-classical MetS biomarkers, the EHCF group had significantly higher aspartate aminotransferase levels (Fig. 3(a)), and CRF was associated with this transaminase $(r=0 \cdot 266 ; P=0 \cdot 002)$. Plasma uric acid (Fig. 3(b)) (a)

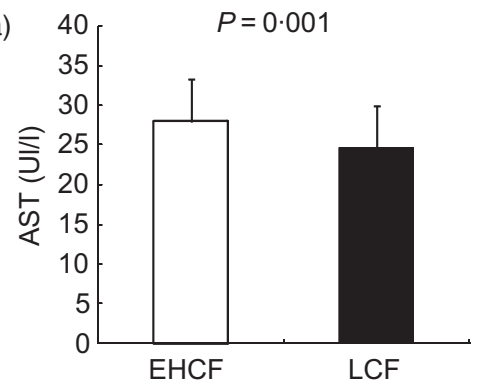

(b) $5.0 \quad P=0.030$

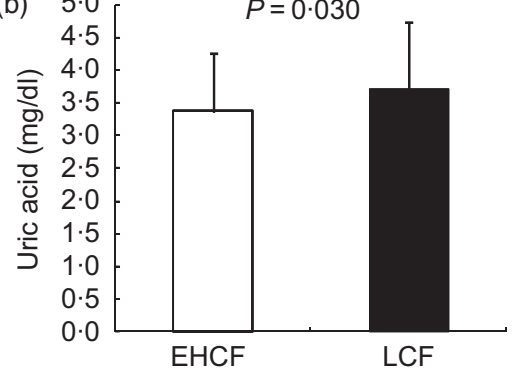

(c)

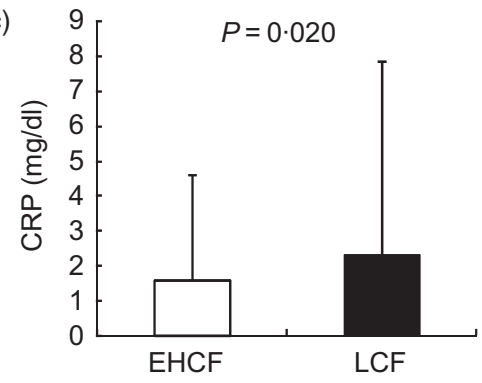

Fig. 3 Non-traditional biomarkers in healthy prepubertal children ( $n$ 141) aged 7-12 years, Córdoba, Spain, according to cardiorespiratory fitness ( $\square$, equal or higher cardiovascular fitness (EHCF) group; $\square$, low cardiovascular fitness (LCF) group): (a) aspartate aminotransferase (AST); (b) uric acid; (c) C-reactive protein (CRP). Values are means and standard deviations represented by vertical bars; $P$ values indicate statistical significance after application of a Student $t$ test to the data

and CRP levels (Fig. 3(c)) were significantly higher in the LCF group than in the EHCF group.

After adjustment for BMI, age and sex, no significant differences were found between groups for the biomarkers analysed. However, using the criteria suggested by Zimmet et al. ${ }^{(4)}, 4 \cdot 25 \%$ of the children were diagnosed as having MetS and all of them belonged to the LCF group. In the correlation analysis, CRF was inversely associated with HOMA-IR $(r=-0 \cdot 240 ; P=0 \cdot 05)$, TAG $(r=-0 \cdot 196 ; P=0 \cdot 023)$, LDL-C $(r=-0 \cdot 227 ; P=0 \cdot 008)$ and uric acid $(r=-0 \cdot 178 ; P=0 \cdot 042)$.

Moreover, when we compared the top and the bottom quintiles after estimating $\mathrm{V}_{\mathrm{O} 2 \mathrm{max}}$ data, we found differences between these groups regarding age, height and systolic and diastolic BP. Children included in the bottom quintile were older and taller and showed higher BP levels than the children in the top quintile, who presented better CRF levels. Apart from the results obtained in 
Table 2 Comparison between the top and bottom quintiles of the studied parameters after estimating $\mathrm{V}_{\mathrm{O} \text { max }}$ : healthy prepubertal children (n 141) aged 7-12 years, Córdoba, Spain

\begin{tabular}{|c|c|c|c|c|c|c|c|}
\hline \multirow[b]{2}{*}{ Variable } & \multicolumn{2}{|c|}{ Quintile 1 ( $n$ 30) } & \multicolumn{2}{|c|}{ Quintile 5 (n 29) } & \multirow[b]{2}{*}{$P^{\star}$} & \multirow[b]{2}{*}{ Typified difference (Cohen $d$ ) } & \multirow[b]{2}{*}{ Effect size } \\
\hline & Mean & SD & Mean & SD & & & \\
\hline Age (years) & $10 \cdot 33$ & 0.96 & $9 \cdot 37$ & $1 \cdot 30$ & 0.002 & 0.84 & 0.39 \\
\hline Weight (kg) & $51 \cdot 72$ & $13 \cdot 73$ & 35.03 & $6 \cdot 29$ & $<0.001$ & 1.56 & 0.62 \\
\hline Height (cm) & $148 \cdot 72$ & $9 \cdot 88$ & $140 \cdot 40$ & $6 \cdot 83$ & $<0.001$ & 0.98 & 0.44 \\
\hline $\mathrm{WC}(\mathrm{cm})$ & $76 \cdot 33$ & $11 \cdot 17$ & $60 \cdot 73$ & $5 \cdot 45$ & $<0.001$ & $1 \cdot 78$ & $0 \cdot 66$ \\
\hline $\mathrm{SBP}(\mathrm{mmHg})$ & $129 \cdot 60$ & $15 \cdot 30$ & 118.03 & $11 \cdot 45$ & 0.002 & $0 \cdot 86$ & 0.39 \\
\hline $\mathrm{DBP}(\mathrm{mmHg})$ & $71 \cdot 27$ & $9 \cdot 23$ & $65 \cdot 14$ & $9 \cdot 61$ & 0.015 & 0.76 & 0.35 \\
\hline BMI $\left(\mathrm{kg} / \mathrm{m}^{2}\right)$ & 23.04 & $4 \cdot 27$ & $17 \cdot 69$ & $2 \cdot 38$ & $<0.001$ & 1.55 & $0 \cdot 61$ \\
\hline $\mathrm{TC}(\mathrm{mg} / \mathrm{dl})$ & $176 \cdot 34$ & $35 \cdot 62$ & $169 \cdot 10$ & $23 \cdot 38$ & $0 \cdot 364$ & 0.24 & $0 \cdot 12$ \\
\hline HDL-C (mg/dl) & $52 \cdot 96$ & $10 \cdot 54$ & $61 \cdot 48$ & $10 \cdot 18$ & 0.003 & -0.82 & -0.38 \\
\hline LDL-C (mg/dl) & $111 \cdot 54$ & $25 \cdot 92$ & $95 \cdot 62$ & $18 \cdot 56$ & $0 \cdot 010$ & $0 \cdot 71$ & 0.33 \\
\hline Apo-A1 (mg/dl) & $139 \cdot 10$ & $18 \cdot 84$ & $152 \cdot 83$ & $15 \cdot 88$ & $0.001^{*}$ & $-0 \cdot 79$ & -0.37 \\
\hline Apo-B $(\mathrm{mg} / \mathrm{dl})$ & $77 \cdot 55$ & $15 \cdot 05$ & $67 \cdot 00$ & $11 \cdot 20$ & 0.004 & $0 \cdot 79$ & 0.37 \\
\hline TAG (mg/dl) & $72 \cdot 43$ & $20 \cdot 53$ & $57 \cdot 96$ & $17 \cdot 38$ & $0.003^{*}$ & $0 \cdot 76$ & 0.35 \\
\hline Glucose (mg/dl) & $82 \cdot 44$ & $7 \cdot 38$ & $82 \cdot 26$ & $6 \cdot 84$ & $0 \cdot 884$ & 0.03 & 0.01 \\
\hline HOMA-IR & $2 \cdot 07$ & $1 \cdot 01$ & 1.05 & 0.58 & $<0.001$ & $1 \cdot 24$ & 0.53 \\
\hline CRP (mg/l) & $1 \cdot 61$ & $1 \cdot 33$ & $1 \cdot 29$ & $1 \cdot 64$ & $0 \cdot 115^{\star}$ & 0.21 & $0 \cdot 11$ \\
\hline AST (UI/I) & $22 \cdot 48$ & $5 \cdot 70$ & $27 \cdot 50$ & $4 \cdot 63$ & $<0.001^{*}$ & -0.97 & -0.44 \\
\hline ALT $(U \mathrm{UI} / \mathrm{I})$ & $22 \cdot 31$ & 28.05 & $17 \cdot 38$ & $6 \cdot 85$ & $0.779^{*}$ & $0 \cdot 24$ & $0 \cdot 12$ \\
\hline Uric acid (mg/dl) & $4 \cdot 02$ & $1 \cdot 13$ & $3 \cdot 15$ & 0.75 & 0.001 & 0.91 & 0.41 \\
\hline
\end{tabular}

Quintile 5, top quintile with the best fitness; quintile 1, bottom quintile with the worst fitness; WC, waist circumference; SBP, systolic blood pressure; DBP, diastolic blood pressure; TC, total cholesterol; HDL-C, HDL cholesterol; LDL-C, LDL cholesterol; HOMA-IR, homeostasis model assessment-insulin resistance index; CRP, C-reactive protein; AST, aspartate aminotransferase; ALT, alanine aminotransferase.

*Statistical significance after application of the Student $t$ test or the Mann-Whitney $U$ test to data expressed as mean and SD; Cohen $d$ and effect size analysis has been done for these groups.

relation to EHCF and LCF groups, we also found higher LDL-C and apo-B levels in the lower quintile (Table 2).

As far as the top and bottom quintiles are concerned, we did not find relevant differences between groups due to the effect size when Cohen's $d$ and effect size correlations were applied. Most of these values were between $0 \cdot 2$ and $0 \cdot 8$ (Table 2), which indicates a moderate effect size.

\section{Discussion}

In the present study, the major finding was that prepubertal children in the LCF group (with low CRF) displayed higher levels for some MetS risk factors such as lipid profile and insulin resistance and for certain non-traditional markers such as uric acid and CRP than did children in the EHCF group. After adjustment for BMI, age and sex, there were no significant between-group differences, suggesting a probable influence of body mass on fitness condition. Moreover, the correlations between traditional and nontraditional risk factors of MetS suggest that individual components of MetS might be influenced by CRF.

Paediatric data are inconsistent regarding whether fitness or fatness is most relevant to health outcomes in children. Only recently, a small number of prospective studies systematically examined the independent and joint associations of CRF and body mass with health outcomes ${ }^{(28)}$. Some research in children suggests that CRF has a protective influence on metabolic health ${ }^{(11,29)}$ whereas other studies report that the effects of fitness may be indirect, mediated through its relationship to fatness ${ }^{(29,30)}$; fatness appears to be more predictive of cardiovascular risk than $\mathrm{CRF}^{(12,13)}$.

In the present study, CRF was assessed using the $20-\mathrm{mSRT}^{(27)}$; since this is a weight-bearing activity, the test might conceivably prompt overestimation of the relationship between $\mathrm{CRF}$ and both fatness and MetS risk factors. In other studies, however, measures were not modified by weight status ${ }^{(10)}$ and others support the validity of using clinical measurements of physical fitness to predict insulin resistance. Low CRF is strongly associated with high BMI and recent investigations suggest that further research is required to explore more fully this relationship ${ }^{(15,27,31)}$. The results obtained in the current study provide additional information about non-traditional metabolic factors in prepubertal healthy children in relation to different levels of CRF and the influence of fatness.

In the present work, children in the EHCF group had lower BMI and a better metabolic profile (lower levels of TAG and higher levels of HDL-C and apo-A1, and lower levels of insulin and HOMA-IR) than did children from the LCF group, suggesting that children with good CRF seem to have better weight status and enjoy better metabolic health. Moreover, this group had lower uric acid and CRP levels. Similarly to our study, higher cardiovascular fitness has been associated with a favourable metabolic profile ${ }^{(12)}$.

The inverse association seems to be consistent with some findings in adolescent studies ${ }^{(32-34)}$ although others do not find these associations with insulin resistance markers ${ }^{(35)}$. The clinical value of fasting glucose as a risk biomarker has been questioned, although in our study there were significant differences between CRF groups. 
In the LCF group, glucose, insulin and HOMA-IR were higher than in the EHCF group, although neither group reached pathological levels (Fig. 2(c)). After adjustment for BMI, however, there were no significant betweengroup differences, suggesting an influence of body mass on glucose metabolism. In a sample of 1140 children, Ruiz et al. ${ }^{(13)}$ found a positive association between these parameters and body fat after adjusting for CRF, and a negative association between both HOMA-IR and fasting insulin and CRF. This inverse association appears to be consistent with findings in adolescent research ${ }^{(32-34)}$ although these associations with insulin resistance markers are not reported in other studies ${ }^{(36)}$. Indeed, HOMAIR has been independently associated with lower CRF in children $^{(15,37,38)}$ and an inverse correlation between HOMA-IR and PA has been also reported ${ }^{(32)}$.

High CRF is associated with low levels of TAG, suggesting that CRF more than PA influences the lipid profile $^{(8,15)}$. Another remarkable result in the present study was that $72 \%$ of the children who performed PA were in the EHCF group. PA has been inversely associated with metabolic risk factors ${ }^{(39)}$ independently of CRF and $\operatorname{adiposity}^{(1,4,11)}$. However, in other studies, its relationship was attenuated $^{(40)}$ or unchanged ${ }^{(41)}$ even after further adjustment according to adiposity ${ }^{(13)}$ and CRF. Research suggests that exercise training improves glucose metabolism $^{(42)}$, inflammation ${ }^{(43)}$ and both lipid and lipoprotein profiles, thus highlighting the impact of PA on cardiovascular risk ${ }^{(44)}$. In our results, the positive significant correlation between PA and CRF may indicate the influence of the different pathways involved in either PA or CRF. PA may thus exert an independent beneficial effect on health regardless of fatness ${ }^{(11)}$.

To study these relationships, several studies have used mixed populations of children and adolescents ${ }^{(45,46)}$, thus ignoring to evaluate the pubertal state. Puberty is associated with a decrease in insulin sensitivity ${ }^{(47)}$; due to this, working only with prepubertal children can reduce this effect. Nevertheless, little research has been done with homogeneous sub-populations ${ }^{(10,11,37)}$. In fact, the low significance in the results of the present study may partly be due to the early age of our groups.

With regard to non-traditional markers, a decline in plasma apo-A1 levels has been associated with low levels of HDL-C, and thus with increased cardiovascular risk ${ }^{(7)}$. In the present study, the LCF group displayed lower levels for these lipid parameters than the EHCF group (Fig. 1). Moreover, although uric acid has been put forward as a new biomarker for metabolic risk in childhood obesity $^{(5,48)}$, its relationship to CRF or PA has not been analysed. Only a study in patients with type 2 diabetes mellitus showed that uric acid was negatively associated with $\mathrm{CRF}^{(49)}$. Children from the LCF group of the present study had higher uric acid levels than EHCF children (Fig. 3(b)), suggesting that CRF may improve this metabolic alteration; a significant negative correlation was also noted between uric acid and CRF. Nevertheless, aspartate aminotransferase was higher in the EHCF group (Fig. 3(a)). The increase in liver transaminases has usually been associated with other components of MetS, particularly insulin resistance; some authors have found a relationship between alanine aminotransferase and CRF or PA in youth $^{(8,34)}$ despite no changes being reported by others ${ }^{(50)}$.

An increase in pro-inflammatory cytokines and acutephase reactants has been associated with cardiovascular risk factors. CRP is an indicator of a range of inflammatory processes of both vascular and non-vascular origin; elevated CRP may reflect a pro-atherogenic metabolic state that predisposes to atherothrombotic events ${ }^{(51)}$. Recent studies in children have shown strong evidence for the association between CRP increase and various markers for MetS ${ }^{(11,52)}$, diabetes mellitus and $\mathrm{CVD}^{(35)}$, and it has been reported that other potential factors and processes such as reduced CRF could contribute to low-grade inflammation in apparently healthy individuals. Here, the EHCF group had lower CRP levels than the LCF group (Fig. 3(c)). In prepubescent children, CRF has been inversely associated with $\mathrm{CRP}^{(43)}$.

Concerning effect size results, there was little or moderate effect size in the present study. Moreover, none of the $P$ values for the parameters compared between top and bottom quintiles groups were near significance. So, perhaps effect size did not have had an important role.

Finally, the International Diabetes Federation consensus report was used to evaluate associations of CRF with MetS, leaving aside the summarized metabolic risk score due to some limitations ${ }^{(4)}$. In a longitudinal study by McMurray et $a l .{ }^{(53)}$, children with MetS (4.6\%) had lower PA levels and CRF outcomes than the non-MetS group. Some $4 \cdot 25 \%$ of prepubertal children in the current study presented MetS; these children belonged to the LCF group and did not perform PA. A similar percentage has been reported in another study of children ${ }^{(1)}$. The classical features for MetS appear to be present even in our general prepubertal paediatric sample and to be related to low CRF. It is therefore important to clarify the impact of $\mathrm{CRF}$ and PA on non-traditional risk factors in children.

In relation to overall public health, new research is needed to evaluate metabolic risk as well as the factors related to it in children with low fitness measured by $\mathrm{V}_{\mathrm{O} \text { max }}$, in different stages and ages. This would contribute to establish the groups with health risk in childhood that could benefit from scheduled PA programmes.

\section{Conclusions}

The metabolic health profile of prepubertal children displaying high levels of CRF is characterized by low TAG, HOMA-IR, uric acid and CRP levels, and higher levels of HDL-C and apo-A1, compared with children with low CRF. The present study provides new information to 
understand the role not only of weight status but also of the level of CRF on the metabolic health profile in prepubertal children in relation to traditional and nontraditional metabolic risk factors.

\section{Acknowledgements}

This research received no specific grant from any funding agency in the public, commercial or not-for-profit sectors. The authors have no conflict of interest. The contribution of each author to the manuscript was as follows: F.J.L.-C., J.d.D.B.-S. and M.G.-d.C. coordinated the fitness and physical activity tests; J.L.P.-N. and M.G.-C. performed the paediatric evaluations; F.J.L.-C. and M.G.-C. processed the biochemical samples; M.C.M.-V. carried out the statistical analyses. All authors contributed to the evaluation of results and writing of the manuscript. Each author has seen and approved the contents of the submitted manuscript.

\section{References}

1. Tailor AM, Peeters PH, Norat T et al. (2010) An update on the prevalence of the metabolic syndrome in children and adolescents. Int J Pediatr Obes 5, 202-213.

2. Weiss R (2010) Metabolic syndrome in childhood - causes and effects. Endocr Dev 19, 62-72.

3. Steele RM, Brage S, Corder K et al. (2008) Physical activity, cardiorespiratory fitness, and the metabolic syndrome in youth. J Appl Physiol 105, 342-351.

4. Zimmet P, Alberti KG, Kaufman F et al.; IDF Consensus Group (2007) The metabolic syndrome in children and adolescents - an IDF consensus report. Pediatr Diabetes $\mathbf{8}$, 299-306.

5. Gil-Campos M, Aguilera CM, Cañete R et al. (2009) Uric acid is associated with features of insulin resistance syndrome in obese children at prepubertal stage. Nutr Hosp 4, 607-613.

6. Bueno G, Bueno O, Moreno LA et al. (2006) Diversity of metabolic syndrome risk factors in obese children and adolescents. Physiol Biochem 62, 125-133.

7. Sellers EA, Singh GR \& Sayers SM (2009) Apo-B/A1 ratio identifies cardiovascular risk in childhood: the Australian Aboriginal Birth Cohort study. Diab Vasc Dis Res 6, 94-99.

8. Bouglé D, Zunquin G, Sesbouë B et al. (2010) Relationships of cardiorespiratory fitness with metabolic risk factors, inflammation, and liver transaminases in overweight youths. Int J Pediatr 580897.

9. Mauras N, Delgiorno C, Kollman C et al. (2010) Obesity without established comorbidities of the metabolic syndrome is associated with a proinflammatory and prothrombotic state, even before the onset of puberty in children. J Clin Endocrinol Metab 95, 1060-1068.

10. Kriemler S, Manser-Wenger S, Zahner L et al. (2008) Reduced cardiorespiratory fitness, low physical activity and an urban environment are independently associated with increased cardiovascular risk in children. Diabetologia 51, 1408-1415.

11. Ekelund U, Anderssen SA, Froberg K et al. (2007) Independent associations of physical activity and cardiorespiratory fitness with metabolic risk factors in children: the European Youth Heart Study. Diabetologia 50, 1832-1840.

12. Froberg K \& Andersen LB (2005) Mini review: physical activity and fitness and its relations to cardiovascular disease risk factors in children. Int J Obes (Lond) 29, Suppl. 2, S34-S39.

13. Ruiz JR, Ortega FB, Rizzo NS et al. (2007) High cardiovascular fitness is associated with low metabolic risk score in children: the European Youth Heart Study. Pediatr Res 61, 350-355.

14. Invitti C, Maffeis C, Gilardini L et al. (2006) Metabolic syndrome in obese Caucasian children: prevalence using WHO-derived criteria and association with nontraditional cardiovascular risk factors. Int J Obes (Lond) 30, 627-633.

15. Suriano K, Curran J, Byrne SM et al. (2010) Fatness, fitness, and increased cardiovascular risk in young children. J Pediatr 157, 552-558.

16. Hasselstrøm H, Hansen SE, Froberg K et al. (2002) Physical fitness and physical activity during adolescence as predictors of cardiovascular disease risk in young adulthood. Danish youth and sports study. An eight-year follow-up study. Int J Sports Med 23, 27-31.

17. Olds T, Tomkinson G, Léger L et al. (2006) Worldwide variation in the performance of children and adolescents: an analysis of 109 studies of the 20-m shuttle run test in 37 countries. Sports Sci 24, 1025-1038.

18. García-Artero E, Ortega FB, Ruiz JR et al. (2007) Lipid and metabolic profiles in adolescents are affected more by physical fitness than physical activity (AVENA study). Rev Esp Cardiol 60, 581-588.

19. McGavock JM, Torrance BD, McGuire KA et al. (2009) Cardiorespiratory fitness and the risk of overweight in youth: the Healthy Hearts Longitudinal Study of Cardiometabolic Health. Obesity (Silver Spring) 17, 1802-1807.

20. Léger LA, Mercier D, Gadoury C et al. (1988) The multistage 20 meter shuttle run test for aerobic fitness. J Sports Sci $\mathbf{6}$, 93-101.

21. Tanner JM (1962) Growth at Adolescence. Oxford: Blackwell.

22. Sobradillo B, Aguirre A, Aresti U et al. (2004) Curvas y tablas de crecimiento (estudios longitudinal y transversal). Madrid: Fundación Faustino Orbegozo Eizaguirre.

23. Moreno LA, Pineda I, Rodríguez G et al. (2002) Waist circumference for the screening of the metabolic syndrome in children. Acta Paediatr 91, 1307-1312.

24. Cole TJ, Bellizzi MC, Flegal KM et al. (2000) Establishing a standard definition for child overweight and obesity worldwide: international survey. BMJ 320, 1240-1243.

25. Pianta RC; National Institute of Child Health and Human Development (2007) Developmental science and education: the NICHD study of early child care and youth development findings from elementary school. Adv Child Dev Behav 35, 253-296.

26. Committee of Experts on Sports Research EUROFIT (1993) Handbook for the EUROFIT Tests of Physical Fitness. Strasburg: Council of Europe.

27. Vale S, Santos R, Soares-Miranda L et al. (2010) The relationship of cardiorespiratory fitness, birth weight and parental BMI on adolescents' obesity status. Eur J Clin Nutr 64, 622-627.

28. LaMonte MJ \& Blair SN (2006) Physical activity, cardiorespiratory fitness, and adiposity: contributions to disease risk. Curr Opin Clin Nutr Metab Care 9, 540-546.

29. Allen DB, Nemeth BA, Clark RR et al. (2007) Fitness is a stronger predictor of fasting insulin levels than fatness in overweight male middle-school children. J Pediatr 150, 383-387.

30. Thomas NE, Cooper SM, Williams SP et al. (2007) Relationship of fitness, fatness, and coronary-heart-disease risk factors in 12- to 13-year-olds. Pediatr Exerc Sc 19, 93-101.

31. Aires L, Silva P, Silva G et al. (2010) Intensity of physical activity, cardiorespiratory fitness, and body mass index in youth. J Phys Act Health 7, 54-59. 
32. Twisk JW, Kemper HC \& van Mechelen W (2002) The relationship between physical fitness and physical activity during adolescence and cardiovascular disease risk factors at adult age. The Amsterdam Growth and Health Longitudinal Study. Int J Sports Med 23, Suppl. 1, S8-S14.

33. Andersen LB, Harro M, Sardinha LB et al. (2006) Physical activity and clustered cardiovascular risk in children: a cross sectional study (The European Youth Heart Study). Lancet 368, 299-304.

34. Kelishadi R, Cook SR, Amra B et al. (2009) Factors associated with insulin resistance and non-alcoholic fatty liver disease among youths. Atherosclerosis 204, 538-543.

35. Shaibi GQ, Ball GD, Cruz ML et al. (2006) Cardiovascular fitness and physical activity in children with and without impaired glucose tolerance. Int J Obes (Lond) 30, 45-49.

36. Katzmarzyk PT, Church TS \& Blair SN (2004) Cardiorespiratory fitness attenuates the effects of the metabolic syndrome on all cause and cardiovascular disease mortality in men. Arch Intern Med 164, 1092-1097.

37. Telford RD, Cunningham RB, Shaw JE et al. (2009) Contrasting longitudinal and cross-sectional relationships between insulin resistance and percentage of body fat, fitness, and physical activity in children - the LOOK study. Pediatr Diabetes 10, 500-507.

38. Puder JJ, Schindler C, Zahner L et al. (2011) Adiposity, fitness and metabolic risk in children: a cross-sectional and longitudinal study. Int J Pediatr Obes 6, e297-e306.

39. Ostojic SM \& Stojanovic MD (2010) High aerobic fitness is associated with lower total and regional adiposity in 12-year-old overweight boys. J Sports Med Phys Fitness 50, 443-449.

40. Rizzo NS, Ruiz JR, Hurtig-Wennlöf A et al. (2007) Relationship of physical activity, fitness, and fatness with clustered metabolic risk in children and adolescents: the European Youth Heart Study. J Pediatr 150, 388-394.

41. Brage S, Wedderkopp N, Ekelund U et al. (2004) Features of the metabolic syndrome are associated with objectively measured physical activity and fitness in Danish children: the European Youth Heart Study (EYHS). Diabetes Care 27, 2141-2148.

42. Pedersen BK \& Saltin B (2006) Evidence for prescribing exercise as therapy in chronic disease. Scand J Med Sci Sports 16, 3-63.
43. Parrett AL, Valentine RJ, Arngrímsson SA et al. (2010) Adiposity, activity, fitness, and C-reactive protein in children. Med Sci Sports Exerc 42, 1981-1986.

44. Ring-Dimitriou S, von Duvillard SP, Paulweber B et al. (2007) Nine months aerobic fitness induced changes on blood lipids and lipoproteins in untrained subjects versus controls. Eur I Appl Physiol 99, 291-299.

45. Anderssen SA, Cooper AR, Riddoch C et al. (2007) Low cardiorespiratory fitness is a strong predictor for clustering of cardiovascular disease risk factors in children independent of country, age and sex. Eur J Cardiovasc Prev Rehabil 14, 526-531.

46. Kwon S, Burns TL \& Janz K (2010) Associations of cardiorespiratory fitness and fatness with cardiovascular risk factors among adolescents: the NHANES 1999-2002. J Phys Act Health 7, 746-753.

47. Hannon TS, Janosky J \& Arslanian SA (2006) Longitudinal study of physiologic insulin resistance and metabolic changes of puberty. Pediatr Res 60, 759-763.

48. Choi HK \& Ford ES (2007) Prevalence of the metabolic syndrome in individuals with hyperuricemia. Am J Med 120, 442-447.

49. Kadoglou NP, Iliadis F, Angelopoulou N et al. (2009) Cardiorespiratory capacity is associated with favourable cardiovascular risk profile in patients with Type 2 diabetes. J Diabetes Complications 23, 160-166.

50. Lee YH, Song YW, Kim HS et al. (2010) The effects of an exercise program on anthropometric, metabolic, and cardiovascular parameters in obese children. Korean Circ J 40, 179-184.

51. Wijnstok NJ, Twisk JW, Young IS et al. (2010) Inflammation markers are associated with cardiovascular diseases risk in adolescents: the Young Hearts project 2000. J Adolesc Health 47, 346-351.

52. Martos R, Valle M, Morales RM et al. (2009) Changes in body mass index are associated with changes in inflammatory and endothelial dysfunction biomarkers in obese prepubertal children after 9 months of body mass index SD score loss. Metabolism 58, 1153-1160.

53. McMurray RG, Bangdiwala SI, Harrell JS et al. (2008) Adolescents with metabolic syndrome have a history of low aerobic fitness and physical activity levels. Dyn Med 7, 5. 